\title{
Activating Transcription Factor 3 Is Essential for Cigarette Smoke-Induced Mucin Expression via Interaction with Activator Protein-1
}

Yan-ping Wu, ${ }^{*}$ Yin-fang Wu, ${ }^{*}$ Chao Zhang, ${ }^{*}$ Hong-bin Zhou, ${ }^{*}$ Chao Cao, ${ }^{*}$ Miao Li, ${ }^{*}$ Chen Zhu, * Song-min Ying, * Zhi-hua Chen, ${ }^{*}$ Hua-hao Shen, ${ }^{* \dagger}$ and Wen $\mathrm{Li}^{*}$

From the Department of Respiratory and Critical Care Medicine,* Second Affiliated Hospital, School of Medicine, Zhejiang University, Hangzhou; and the State Key Lab of Respiratory Disease, ${ }^{\dagger}$ Key Site of National Clinical Research Center for Respiratory Disease, Guangzhou, China

Accepted for publication October 4, 2016.

Address correspondence to Wen Li, Ph.D., M.D., Hua-hao Shen, Ph.D., M.D., or Zhi-hua Chen, Ph.D., Department of Respiratory and Critical Care Medicine, Second Affiliated Hospital, School of Medicine, Zhejiang University, 88 Jiefang Rd., Hangzhou 310009,

China. E-mail:

zhihuachen2010@163.com, huahaoshen@163.com,or liwenhz@163.com.

\begin{abstract}
Mucus hypersecretion is an important pathologic feature of chronic obstructive pulmonary disease. Activating transcription factor 3 (ATF3) is an adaptive-response gene that participates in various cellular processes. However, little is known about its role in cigarette smoke (CS)-induced mucus hyperproduction. This study aimed to investigate the role and molecular mechanisms of ATF3 in CS-induced Mucin 5AC (MUC5AC) expression. ATF3 was elevated in lung tissues of mice exposed to CS for 12 weeks. Treatment with CS extract significantly induced ATF3 expression and MUC5AC production in human bronchial epithelial cells, NCI-H292, and mouse tracheal epithelial cells. Interference of ATF3 significantly attenuated CS-induced MUC5AC expression in NCI-H292 and human bronchial epithelial cells. Mouse tracheal epithelial cells isolated from $A t f 3^{-/-}$mice also exhibited less MUC5AC production in response to CS extract treatment. In vivo, the $A t f 3^{-/-}$mice also displayed a significantly reduced mucus production relative to wild-type controls in response to chronic CS exposure. Furthermore, a chromatin immunoprecipitation assay revealed increased ATF3 binding to the MUC5AC promoter after CS treatment, and this transcriptional binding was significantly inhibited by knockdown of JUN, a subunit of activator protein-1. These results demonstrate that ATF3 may be involved in activator protein-1 signaling and transcriptional promotion of CS-induced MUC5AC expression in airway epithelial cells. (Am J Pathol 2017, 187: 280-291; http://dx.doi.org/10.1016/j.ajpath.2016.10.012)
\end{abstract}

Chronic obstructive pulmonary disease (COPD) is a disease characterized by a continuous limitation of airflow that is not fully reversible. ${ }^{1}$ The worldwide rate of mortality and morbidity of this disease has increased rapidly in recent decades. According to World Health Organization reports, 65 million people are estimated to have moderate-to-severe COPD (http://www.who.int/respiratory/copd/burden/en). By 2010, COPD was reported to be the third leading cause of death. ${ }^{2}$ In 2012, >3 million people died of COPD, which accounted for $6 \%$ of global mortality that year (http://www. who.int/mediacentre/factsheets/fs315/en).

Excessive airway mucus secretion is a major pathophysiological feature in all COPD patients. ${ }^{3}$ Mucus is composed of various kinds of proteins, among which mucins are the primary secretory components produced by the airway epithelium. As the major mucin in the respiratory tract, the secreted gel-forming protein Mucin 5AC (MUC5AC) is considered to be pivotal in COPD pathogenesis. The persistent accumulation of airway mucus can provide an environment that supports microbial growth and multiplication, resulting in infection and inflammation.

Chronic exposure to environmental irritants, such as cigarette smoke (CS), occupational dusts and chemicals, and indoor or outdoor air pollution, can damage the lungs and the airways, which is usually considered to be a cause of COPD. ${ }^{4}$ More than $80 \%$ of patients with COPD are cigarette

Supported by National Natural Science Foundation of China grants 81170037 and 81370126 (W.L.) and the project from the National Clinical Research Center of China for Respiratory Disease.

Y.-p.W. and Y.-f.W. contributed equally to this work.

Disclosures: None declared. 
smokers. ${ }^{5} \mathrm{CS}$ is established as a major commonly encountered risk factor for the development of $\mathrm{COPD}^{6}$ and is closely related to mucus overproduction. ${ }^{7}$ It is now recognized that mucus hyperproduction in COPD patients contributes to airway obstruction, the accelerated reduction of lung function, morbidity, ${ }^{8,9}$ and mortality. ${ }^{10,11}$ Thus, targeting CS-induced mucus hypersecretion appears to be a promising strategy for COPD treatment. However, the molecular mechanisms that underlie CS-associated mucus dysfunction are not fully understood.

Activating transcription factor 3 (ATF3) is a member of the ATF/cyclic AMP response element-binding (CREB) family of basic leucine zipper (bZip) transcription factors. ${ }^{12}$ As an adaptive-response gene, ATF3 is involved in cellular processes that allow cells to adapt to extracellular and/or intracellular stimuli. ${ }^{12}$ ATF3 forms homodimers or heterodimers with bZip proteins, such as the activator protein-1 (AP-1), CCAAT-enhancer-binding protein, or musculoaponeurotic fibrosarcoma families. ${ }^{13}$ These heterodimers can act as either repressors or activators of transcription, depending on the promoter context, ${ }^{14}$ and the existence of other ATF/CREB family members may ultimately decide the role of ATF3 in transcriptional regulation. ${ }^{15}$

The role of ATF3 in COPD pathogenesis remains unknown. It has been confirmed that AP-1 activation and its binding sites in the MUC5AC promoter mediates the positive regulation of CS-induced MUC5AC transcription in airway epithelial cells. ${ }^{16}$ Because ATF3 can interact with AP-1, we hypothesized that it might regulate CS-induced MUC5AC expression via the AP-1 family. To test this hypothesis, we performed a series of in vitro and in vivo experiments and revealed that ATF3 up-regulates MUC5AC expression through ATF3/AP-1 complex-mediated transcriptional activation in airway epithelial cells.

\section{Materials and Methods}

\section{Animal Models}

Atf $3^{-/-}$mice were kindly provided by Tsonwin Hai Laboratory (The Ohio State University, Columbus, $\mathrm{OH}$ ). Wildtype (WT) C57BL/6 controls were purchased from the Laboratory Animal Center of Zhejiang University. At 6 to 8 weeks of age, male Atf $3^{-1-}$ and WT mice were randomly assigned to the experimental or control groups and raised in isolated cages. The experimental group was exposed to the smoke generated from 100 research cigarettes via a smoking machine every day (TE-10; Teague Enterprises, Woodland, CA) for 12 weeks (5 days per week). The total particulate matter concentration in the chamber was approximately 160 to $180 \mathrm{mg} / \mathrm{m}^{3}$. Accordingly, the control group was exposed to filtered air. All animal experimental protocols were approved by the Ethical Committee for Animal Studies of Zhejiang University in China. In addition, we performed the methods in accordance with approved guidelines.

\section{Cell Line and Cell Culture}

Human bronchial epithelial (HBE) cells and human NCIH292 cells (lung mucoepidermoid carcinoma cell line) were purchased from the ATCC (Manassas, VA), propagated and maintained in RPMI 1640 medium supplemented with $10 \%$ fetal bovine serum in a $37^{\circ} \mathrm{C}, 5 \% \mathrm{CO}_{2}$ incubator. After reaching $>90 \%$ confluence, the cells were incubated in serum-free medium for 12 hours to synchronize the cell cycle and to return serum-activated signaling pathways to basal levels before exposure to CS extract (CSE).

To isolate and identify airway epithelial cell-specific response in vitro, primary mouse tracheal epithelial cells (MTECs) were obtained from the tracheas of C57BL/6 mice or $A t f 3^{-1-}$ mice. Harvesting of MTECs was performed in accordance with previously described methods, ${ }^{17}$ and the cells were seeded onto Transwell inserts in 12-well plates (0.4-mm pore, 12-mm diameter; Corning, Corning, NY) and cultured at $37^{\circ} \mathrm{C}$ in a $5 \% \mathrm{CO}_{2}$ incubator. After an approximately 2-week proliferative phase, the cells reached confluence. The apical medium was removed to switch the cells to the differentiation phase (an additional 2 weeks) at an air-liquid interface (ALI) to achieve mucociliary differentiation. The cells were then exposed to CSE after differentiation was established.

\section{Reagents}

Antibodies specific for actin beta (ACTB), ATF3, FOS, and JUN were purchased from Santa Cruz Biotechnology (Dallas, TX). Antibodies specific for phospho-FOS (pFOS) and phospho-JUN (p-JUN) were purchased from Cell Signaling Technology (Danvers, MA). Antibody specific for MUC5AC was purchased from Abcam (Cambridge, MA). All siRNAs were purchased from Santa Cruz Biotechnology. SP600125 was purchased from Sigma-Aldrich (St. Louis, MO).

\section{CSE}

In vitro, aqueous CSE was used to imitate the effects of CS smoke. Smoke from one standard research cigarette (code $2 \mathrm{R} 1 \mathrm{~F}$, from the University of Kentucky Tobacco Research Institute) was slowly bubbled through $10 \mathrm{~mL}$ of RPMI 1640 medium at a speed of 5 minutes per cigarette. The CSE solution was regarded as $100 \%$. The samples were then filtered through a $0.22 \mu \mathrm{mol} / \mathrm{L}$ Millex-GS (Millipore, Watford, United Kingdom) filter, frozen in aliquots, and stored at $-80^{\circ} \mathrm{C}$. An aliquot of CSE was thawed immediately before use.

\section{siRNA/Plasmid Studies}

After reaching 50\% confluence, $\mathrm{HBE}$ and NCI-H292 cells were transfected with siRNA at a concentration of $5 \mathrm{nmol} / \mathrm{L}$ 
with GeneMute TM (SignaGen Laboratories, Rockville, MD). The cells were transfected with $2.5 \mu \mathrm{g}$ of plasmid with Polyget (SignaGen Laboratories) when they reached $90 \%$ confluence, according to the manufacturer's protocols. Twenty-four hours after siRNA transfection and 12 hours after plasmid transfection, the transfected cells were serumstarved for 12 hours and then exposed to CSE.

\section{Inhibitor Studies}

HBE cells were serum-starved for 12 hours and exposed to the indicated concentration of SP600125, which is a specific inhibitor for Jun amino-terminal kinase 1/2 mitogenactivated protein kinase. After 30 minutes, the cells were exposed to 3\% CSE for 24 hours. The MUC5AC mRNA expression level was detected.

\section{qPCR Analysis}

The cells were harvested, and total RNA was extracted with the TRIzol reagent (Invitrogen, Carlsbad, CA). Equal amounts of total RNA were reverse-transcribed into cDNA using the PrimerScript RT Reagent Kit (Takara Bio Inc., Otsu, Japan) according to the manufacturer's instructions. For real-time quantitative PCR (qPCR), the SYBR Green PCR Master Mix (Takara Bio Inc.) and a CFX96quantified PCR system (Bio-Rad, Hercules, CA) were used according to the manufacturer's protocols. Relative expression was calculated using the $2^{-\Delta \Delta C T}$ method. The sequences of the primers used for amplification are listed in Table 1.

\section{Western Blot Analysis}

Total cell lysates were obtained in radioimmunoprecipitation assay buffer supplemented with a cocktail of protease inhibitors and phosphatase inhibitors. Equal amounts of each sample were separated by SDSPAGE and transferred to polyvinylidene difluoride membranes (Bio-Rad). The membranes were incubated with the antibodies described above overnight and were then probed with secondary antibodies. The immune complexes were visualized with a Western blot detection system (Odyssey; Li-COR Bioscience, Lincoln, NE).

\section{Histology and Immunohistochemistry}

Seven to eight mice from each group were sacrificed 1 day after the last smoke challenge, and the left lungs were inflated and fixed with an intratracheal injection of $4 \%$ paraformaldehyde in phosphate-buffered saline (PBS). The lungs were then immersed in the same fixative to preserve the pulmonary architecture. The tissues were embedded in paraffin, cut into sections at a thickness of $4 \mu \mathrm{m}$, and mounted onto slides for immunohistochemistry staining of MUC5AC and periodic acid-Schiff to estimate mucus production.

\section{Immunofluorescence Analysis}

HBE cells were seeded and cultured on coverslips (Fisher Scientific Co., Pittsburgh, PA). After 12 hours in serumstarved conditions, the cells were exposed to $3 \%$ CSE for various periods of time. The cells were washed with PBS and fixed with $4 \%$ formaldehyde for 30 minutes. The cells were blocked with $5 \%$ bovine serum albumin in PBS for 30 minutes at room temperature, then incubated with antibodies overnight at $4^{\circ} \mathrm{C}$. The coverslips were then washed and incubated with fluorescently labeled secondary antibody for 1 hour at room temperature. Finally, the nuclei were stained with DAPI to identify cells.

\section{Co-Immunoprecipitation}

Equivalent amounts of nuclear protein were immunoprecipitated in a lysis buffer containing an antibody specific for FOS or p-FOS and protein A-agarose (Santa Cruz Biotechnology) at $4{ }^{\circ} \mathrm{C}$ overnight. The precipitates were collected after washing three times with lysis buffer, and ATF3 and AP-1 (including FOS, JUN, p-FOS, and p-JUN) protein levels were detected by Western blot analysis, as described above.

\section{Luciferase Activity Assay}

The MUC5AC-luciferase plasmid was kindly provided by Jian-dong Li (Georgia State University, Atlanta, GA). The MUC5AC gene promoter PCR product was inserted into the firefly luciferase reporter vector pGL3-basic. HBE cells

Table 1 Primer Sets for RT-PCR Analysis and ChIP Assay

\begin{tabular}{|c|c|c|c|}
\hline Genes & Forward & Reverse & Assay \\
\hline $\operatorname{MUC5AC}(\mathrm{h})$ & 5'-CAGCACAACCCCTGTTTCAAA-3' & 5'-GCGCACAGAGGATGACAGT-3' & RT-PCR \\
\hline $\operatorname{Muc5ac}(\mathrm{m})$ & 5'-CTGTGACATTATCCCATAAGCCC-3' & $5^{\prime}-$ AAGGGGTATAGCTGGCCTGA-3' & RT-PCR \\
\hline ATF3 (h) & 5'-GAGGATTTTGCTAACCTGACGC-3' & $5^{\prime}$-CTACCTCGGCTTTTGTGATGG-3' & RT-PCR \\
\hline Atf3 (m) & $5^{\prime}$-GAGGATTTTGCTAACCTGACACC-3' & $5^{\prime}-$ TTGACGGTAACTGACTCCAGC- $3^{\prime}$ & RT-PCR \\
\hline$A C T B(h)$ & $5^{\prime}$-CATGTACGTTGCTATCCAGGC-3' & $5^{\prime}$-СTCСTTAATGTCACGCACGAT-3' & RT-PCR \\
\hline
\end{tabular}

ChIP, chromatin immunoprecipitation; h, human; $m$, mouse. 
were seeded in 24-well plates and allowed to adhere overnight. The control or ATF3 plasmid was cotransfected into cells with a mixture of the MUC5AC-promoter plasmid and the internal control Renilla luciferase reporter pRL-TK. Forty-eight hours after transfection, the cells were recovered in serum-free medium for 12 hours and then exposed to $1 \%$ CSE for 24 hours. For experiments with siRNA, HBE cells were pretreated with control or ATF3 siRNA. Twenty-four hours later, the cells were transfected with the MUC5AC-luciferase plasmid and then treated with CSE. The cells were harvested for subsequent analysis, and dual luciferase assays were performed according to the manufacturer's instructions (Promega, Madison, WI). The values for firefly luciferase were normalized to Renilla luciferase under the control of the pRL-TK vector.

\section{ChIP Analysis}

Chromatin immunoprecipitation (ChIP) was performed according to the manufacturers' instructions (ChIP Assay Kit; Upstate Biotechnology, Waltham, MA). After CSE stimulation, HBE cells were fixed with $1 \%$ formaldehyde at room temperature for 10 minutes and terminated with glycine. The fixed cells were harvested with ice-cold PBS containing Protease Inhibitor Cocktail II, and the pellet was resuspended in SDS lysis buffer. Then, the DNA was sheared to 200- to
A

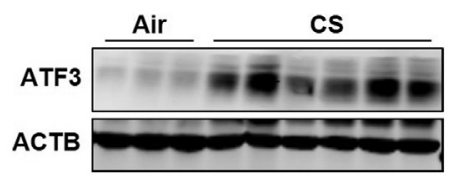

B

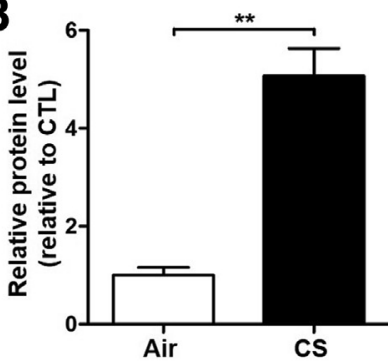

E

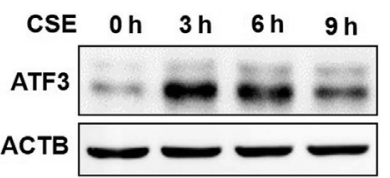

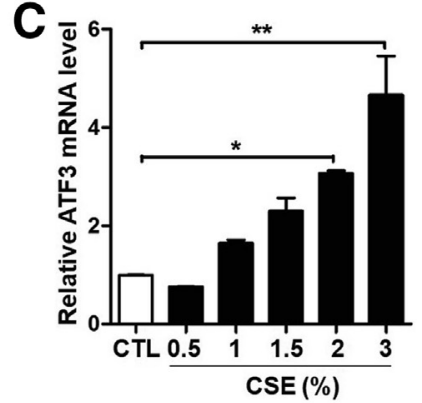

$\mathbf{F}$

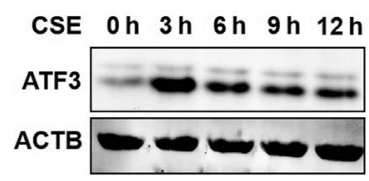

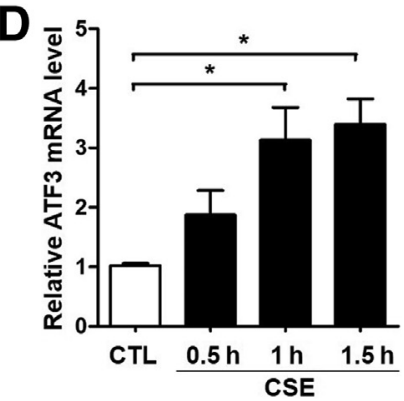

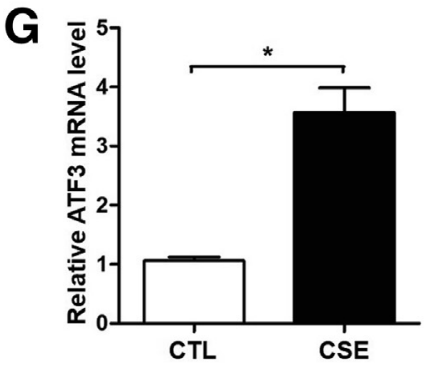

H
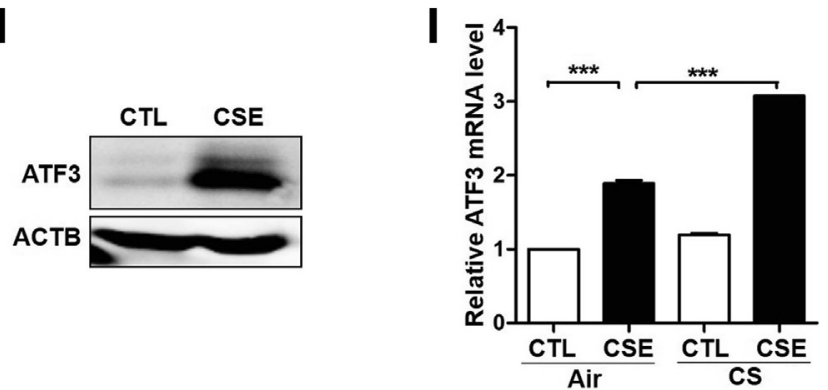

Figure 1 CS stimulates ATF3 expression in lung tissues and airway epithelial cells. A: C57BL/6 mice were exposed to CS for 12 weeks. Lungs were isolated from individual mice 1 day after the last smoke challenge, and protein expression of ATF3 was analyzed by Western blot analysis. B: Quantification of the bands presented in A. C-E: Human bronchial epithelial cells were grown in submerged cell culture conditions until confluent and treated with increasing concentration of CSE for 1.5 hours (C) or 3\% CSE for the indicated duration before harvest (D and E). Equal amounts of RNA and protein from total cell extracts were analyzed for ATF3. F: NCI-H292 cells were grown in submerged cell culture conditions until confluent and treated with $3 \%$ CSE for the indicated duration before harvest. Equal amounts of protein from total cell extracts were analyzed for ATF3. $\mathbf{G}$ and $\mathbf{H}$ : The expression of ATF3 mRNA and protein in well-differentiated MTECs were treated with 1\% CSE for 12 hours. I: MTECs were obtained from the tracheas of C57BL/6 mice exposed to CS for 4 weeks, then subjected to $1 \%$ CSE stimulation in ALI condition. Gene expression analysis for ATF3 was detected by qPCR. Data are expressed as means \pm SEM. $n=3$ independent experiments; CTL, $n=3$ CTL mice (A); $n=6$ mice for CS exposure (A). An unpaired two-tailed $t$-test was used in B and G. One-way analysis of variance followed by Newman-Keuls test was used in C, D, and I. ${ }^{*} P<0.05,{ }^{* *} P<0.01$, and ${ }^{* * *} P<0.001$. ALI, air-liquid interface; ATF3, activating transcription factor 3; CS, cigarette smoke; CSE, cigarette smoke extract; CTL, control; MTECS, mouse tracheal epithelial cells; qPCR, real-time quantitative PCR. 
1000-bp fragments by sonication. Immunoprecipitation was performed using an anti-ATF3 antibody with rotation overnight at $4^{\circ} \mathrm{C}$. Protein/DNA complexes were captured in elution buffer, and cross-links were reversed to free DNA. After DNA purification, quantitative PCR was performed using $M U C 5 A C$ promoter-specific primers (Table 1).

\section{Statistical Analysis}

Experimental data were quantified as the means \pm SEM. The results were analyzed using Prism version 5 (GraphPad Inc., San Diego, CA). Comparisons between two groups were evaluated by using an unpaired two-tailed $t$-test. For multiple groups, one-way analysis of variance followed by the Newman-Keuls test was used. $P$ values $<0.05$ were considered to indicate statistical significance.

\section{Results}

\section{CS Induces ATF3 Expression in Lung Epithelial Cells}

The level of ATF3 in CS-exposed lung tissues was measured by Western blot analysis. C57BL/6 mice were exposed to CS or filtered air for 12 weeks. ATF3 levels were increased in the lungs of CS-exposed mice in comparison with the air controls (Figure 1, A and B).

In addition, Western blot and qPCR analyses confirmed that ATF3 levels were elevated in dose- and time-dependent
A

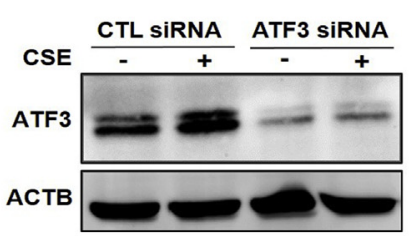

C

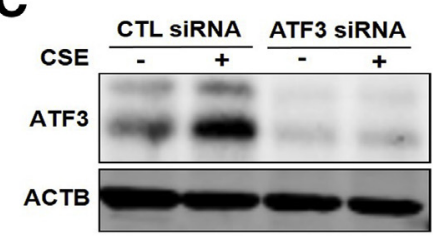

E

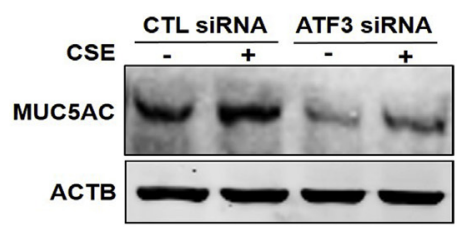

G

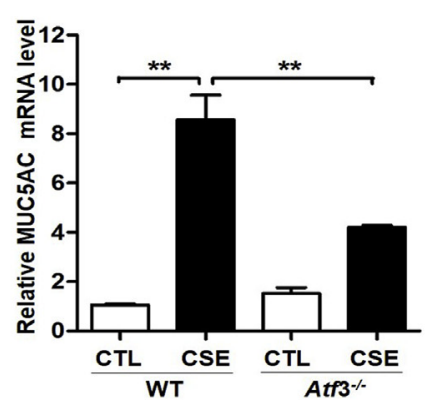

B

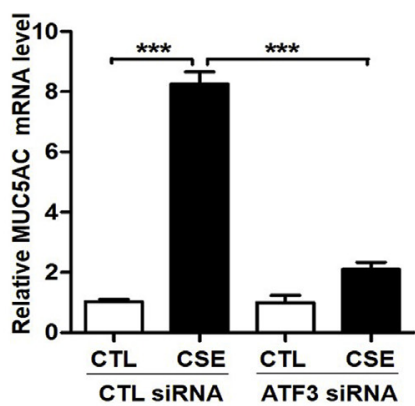

D

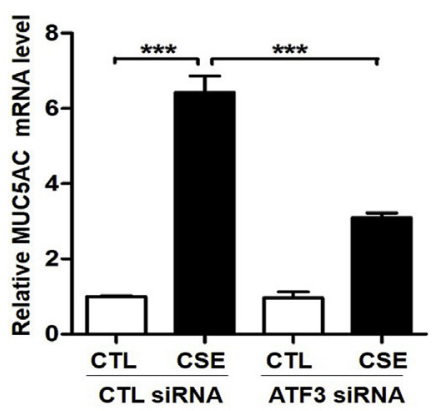

$\mathbf{F}$

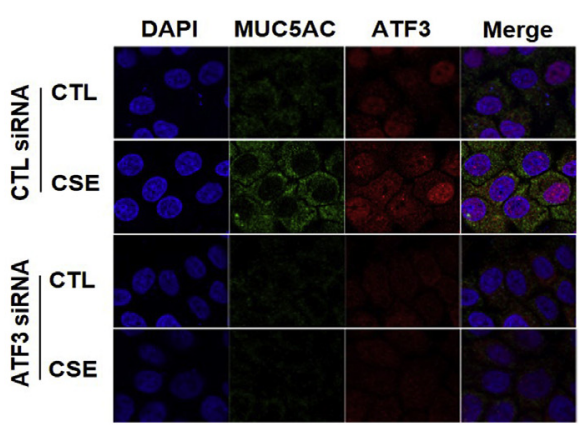

Figure 2 Effects of ATF3 gene silencing on CSEinduced MUC5AC expression. Cells were transfected with $5 \mathrm{nmol} / \mathrm{L}$ CTL or ATF3 siRNA and then stimulated by $3 \%$ CSE for an additional 24 hours. A and C: The efficiency of transfection is examined by Western blot analysis in NCI-H292 (A) and HBE cells (C). B and D: The relative level of MUC5AC mRNA transcript was assessed by qPCR in NCI-H292 and HBE cells, respectively. E: The protein level of MUC5AC in HBE cells was measured by Western blot analysis. F: Representative immunofluorescence images of MUC5AC in HBE cells. DAPI counterstain was used to identify cell nucleus. G: MTECs were extracted from WT and $\mathrm{Atf3}^{-/-}$mice and then exposed to 1\% CSE for 24 hours in an ALI culture. The relative level of MUC5AC mRNA transcript was assessed by qPCR. Data are expressed as means \pm SEM. $n=3$ independent experiments. One-way analysis of variance followed by Newman -Keuls test was used on all comparisons. ${ }^{* *} P<0.01,{ }^{* * *} P<0.001$. ALI, air-liquid interface; ATF3, activating transcription factor 3 ; CSE, cigarette smoke extract; CTL, control; HBE, human bronchial epithelial; MTECs, mouse tracheal epithelial cells; MUC5AC, Mucin 5AC; qPCR, realtime quantitative PCR; WT, wild-type. 
manners on CSE exposure in HBE cells (Figure 1, C-E) and NCI-H292 cells (Figure 1F). We further verified the ability of CSE to induce ATF3 expression in welldifferentiated MTECs. We used an established protocol to isolate epithelial cells and to culture the cells in ALI conditions, as described in Materials and Methods. In MTECs exposed to CSE, ATF3 was induced significantly at both the mRNA and protein levels (Figure 1, G and $\mathrm{H}$ ). Moreover, Atf3 level was induced more significantly in MTECs from CS-exposed mice than in MTECs from air-treated controls (Figure 1I), suggesting that MTECs from CS-exposed mice are more sensitive to CSE stimulation.

\section{ATF3 Silencing Decreases CSE-Induced MUC5AC Expression in Vitro}

ATF3 has been demonstrated to dampen goblet cell hyperplasia and mucus hypersecretion in a mouse asthma model. ${ }^{18}$ We thus evaluated the potential role of ATF3 in the regulation of CS-induced mucus expression.
We first silenced ATF3 using targeted siRNA (Figure 2, $\mathrm{A}$ and $\mathrm{C}$ ) and measured the transcript abundance of MUC5AC in HBE and NCI-H292 cells. The gene expression and protein production of MUC5AC were assessed by qPCR, Western blot analysis, or immunofluorescence. CSEinduced MUC5AC was significantly inhibited by ATF3 siRNA (Figure 2, B and D-F). To confirm these results, MTECs from $A t f 3^{-1-}$ and WT mice were exposed to $1 \%$ CSE in the ALI culture system. Consistently, the lack of the Atf3 gene notably attenuated CSE-induced Muc5ac mRNA expression in MTECs (Figure 2G). All of these data suggest that ATF3 is essential for CSE-induced MUC5AC expression in airway epithelial cells.

\section{ATF3 Deficiency Relieves CS-Stimulated Airway MUC5AC Expression in Vivo}

We further evaluated the effect of ATF3 on the expression of MUC5AC in the CS-exposed murine model. WT or $A t f 3^{-1-}$ mice were exposed to CS for 12 weeks. As shown
A

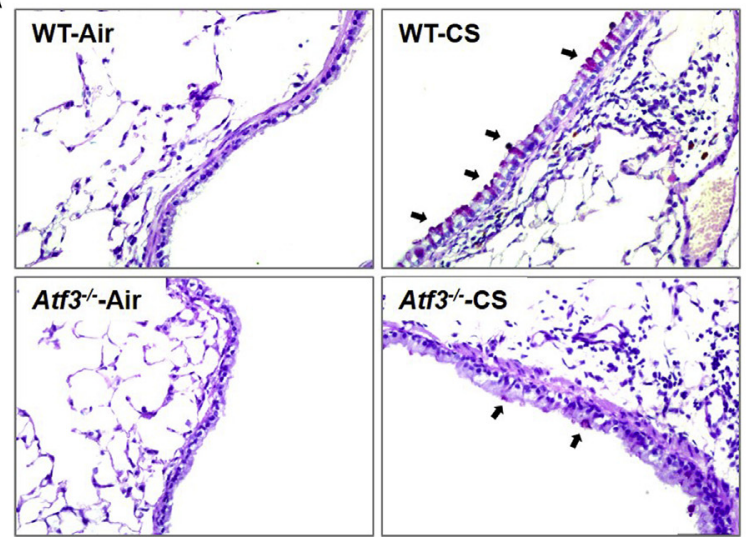

C

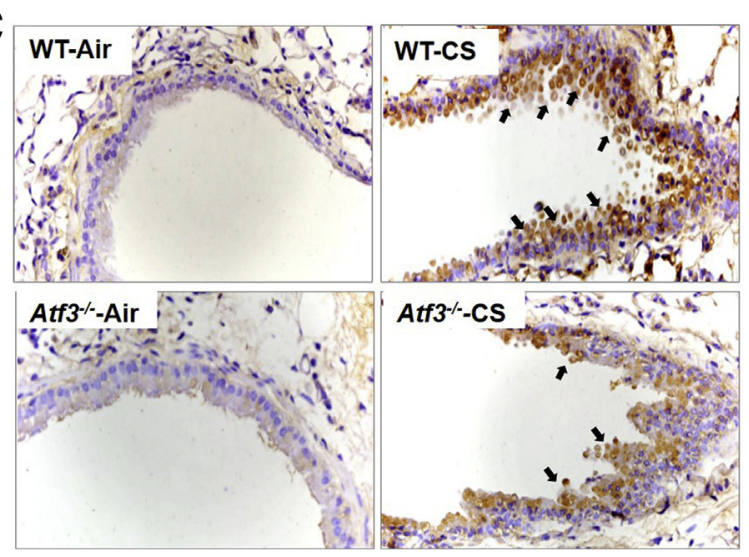

B

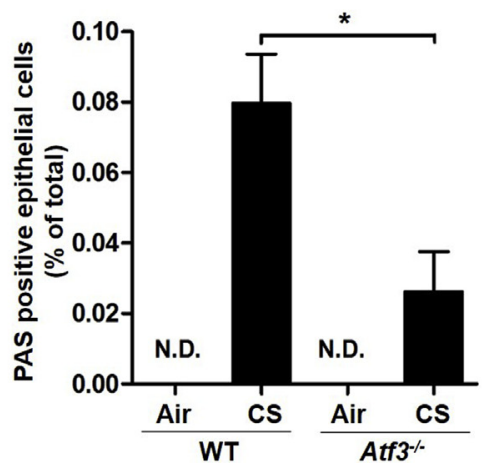

D

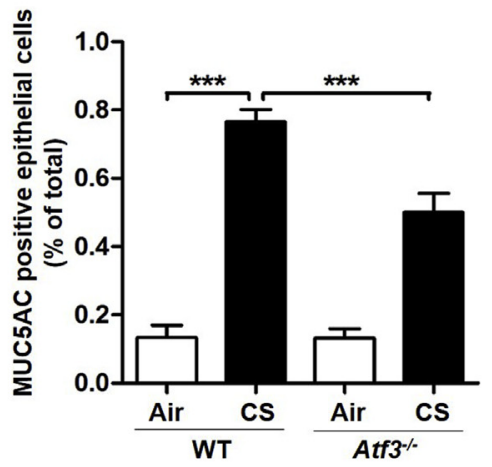

Figure $3 \mathrm{Atf3^{-/- }}$ mice display decreased airway mucus production, and MUC5AC expression in response to CS exposure. Atf $3^{-/-}$and WT mice were exposed to CS for 12 weeks. Lungs were isolated 1 day after the last smoke challenge. A: Representative images of lung sections by PAS staining. B: Quantified percentage of PAS-positive cells in the epithelium. C: Representative images of lung sections stained for MUC5AC. Arrows in A and C point to PAS-stained goblet cells and MUC5AC-positive staining of the airway epithelium, respectively. D: Quantified percentage of MUC5AC-positive cells in the epithelium. Data are expressed as means \pm SEM. $n=7$ to 8 mice for each group. An unpaired two-tailed $t$-test was used in B. One-way analysis of variance followed by NewmanKeuls test was used in D. ${ }^{*} P<0.05,{ }^{* *} P<0.001$. ATF3, activating transcription factor 3; CS, cigarette smoke; MUC5AC, Mucin 5AC; N.D., not detectable; PAS, periodic acid-Schiff; WT, wild-type. 
in Figure 3, A and B, goblet cells were undetectable in the airways of control mice but were evident in CS-exposed WT mice. As expected, CS-treated Atf $3^{-/-}$mice showed decreased goblet cells in comparison with WT controls. In addition, the large airways of WT mice exhibited increased expression of MUC5AC, whereas the CS-treated Atf $3^{-1-}$ mice exhibited a significant reduction of MUC5AC expression, as evidenced by immunohistochemistry staining (Figure 3C). Quantification of the percentage of MUC5AC-positive cells in the epithelium showed stronger MUC5AC staining in the airways of CS-exposed WT mice than $A t f 3^{-1-}$ mice (Figure 3D). Consistent with the in vitro results shown in Figure 2, these findings demonstrate that ATF3 is a positive regulator of CS-induced MUC5AC expression in vivo.

\section{ATF3 Overexpression Exerts No Effect on CSE-Induced MUC5AC Expression}

The deficiency of the ATF3 gene has been shown to reduce CSE-stimulated MUC5AC expression. Thus, we sought to explore whether overexpression of the ATF3 gene would promote CSE-induced MUC5AC production. To address this question, an ATF3 expression vector was transfected into NCI-H292 and HBE cells (Figure 4, A and C). Interestingly, increased exogenous ATF3 expression exerted no effect on basal or CSE-induced MUC5AC expression in airway epithelial cells (Figure 4, B and D).
These data suggest that CSE-induced endogenous ATF3 expression is sufficient for the regulation of MUC5AC expression.

\section{AP-1 Is Involved in CSE-Induced MUC5AC Expression}

Previous research demonstrated that AP-1 activation is required for CSE-induced mucin production, ${ }^{16}$ so we detected AP-1 expression in the CS exposure model both in vivo and in vitro. After exposed to CS for 12 weeks, the lungs of CS-treated WT mice displayed elevated levels of the AP-1 subunits FOS and JUN and their phosphorylated forms (Figure 5, A and B). In vitro, AP-1-related proteins were all increased in a time-dependent manner and peaked after 9 hours of 3\% CSE exposure (Figure 5C). Furthermore, treatment with either JUN siRNA or the Jun aminoterminal kinase inhibitor SP600125 significantly decreased the expression of MUC5AC (Figure 5, D and E). These results demonstrate that AP-1 is involved in CSE-induced MUC5AC production in airway epithelial cells.

\section{CS Activates the ATF3/AP-1 Signaling Pathways}

Because both ATF3 and AP-1 were demonstrated to positively regulate CS-induced mucin production, we next sought to explore whether any crosstalk occurs between these two pathways. Genetic analysis revealed that no direct ATF3 binding sites exist in the MUC5AC promoter.

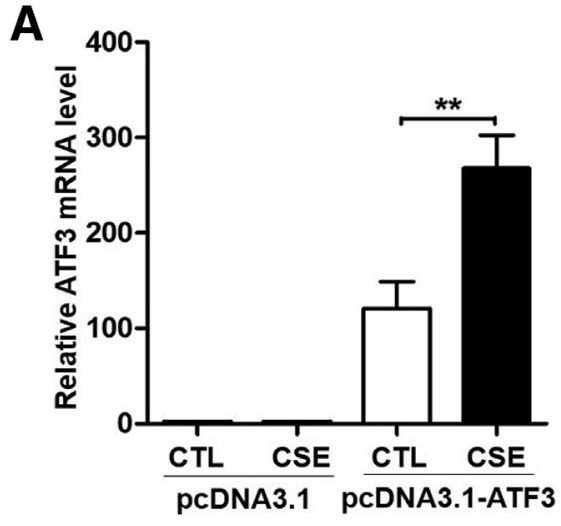

C

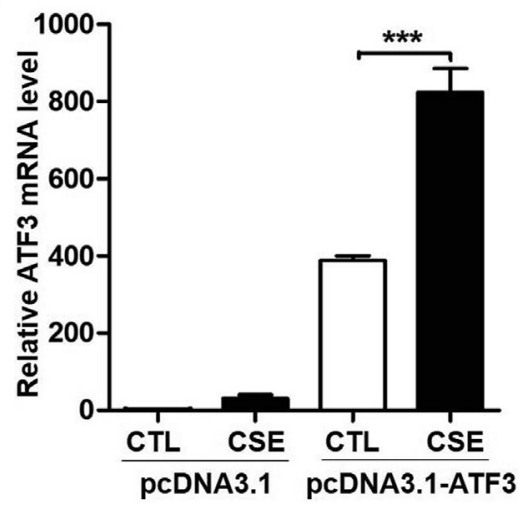

B

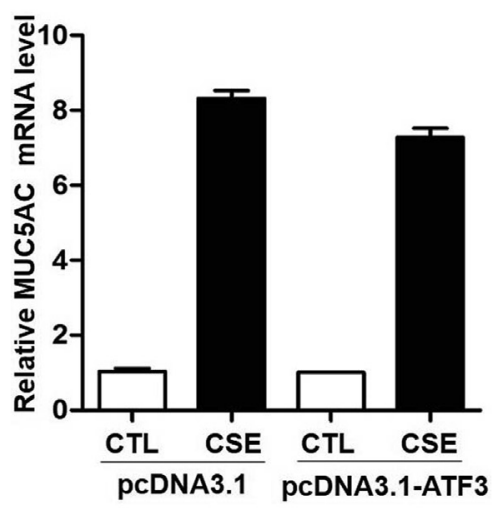

D

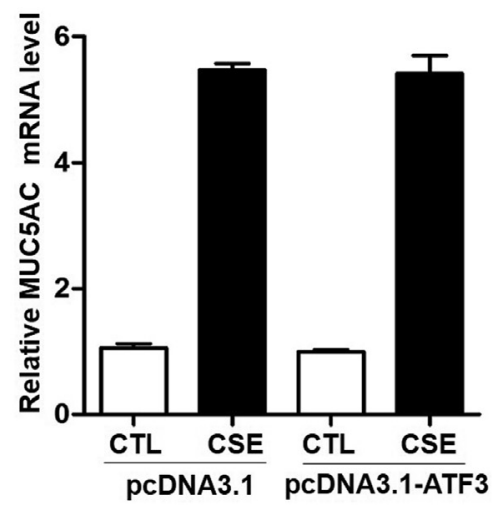

Figure 4 Effects of ATF3 gene overexpression on CSE-induced MUC5AC expression. Cells were transfected with $2.5 \mu \mathrm{g}$ CTL or ATF3 plasmid and then stimulated with $3 \%$ CSE for an additional 24 hours. The relative levels of ATF3 and MUC5AC mRNA transcripts were assessed by qPCR in NCIH292 cells (A and B) and human bronchial epithelial cells (C and $\mathbf{D})$. Data are expressed as means \pm SEM. $n=3$ independent experiments. One-way analysis of variance followed by NewmanKeuls test was used on all comparisons. ${ }^{* * P}<0.01,{ }^{* * * P}<0.001$. ATF3, activating transcription factor 3 ; CSE, cigarette smoke extract; CTL, control; MUC5AC, Mucin 5AC; pcDNA3.1, control plasmid; qPCR, real-time quantitative PCR. 

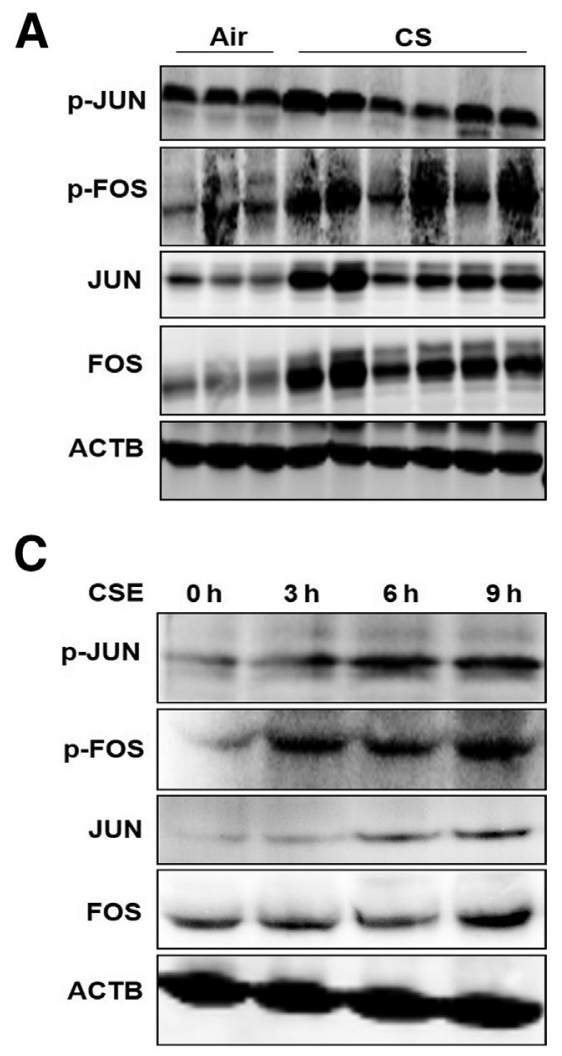

E

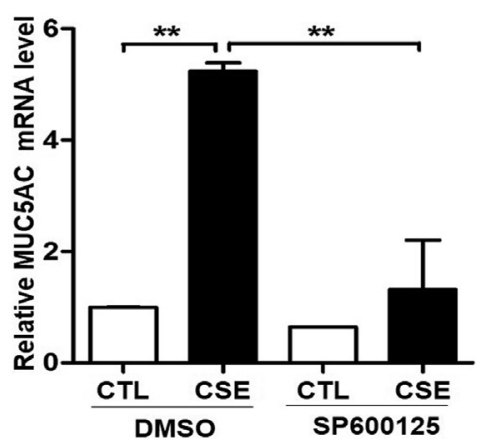

B

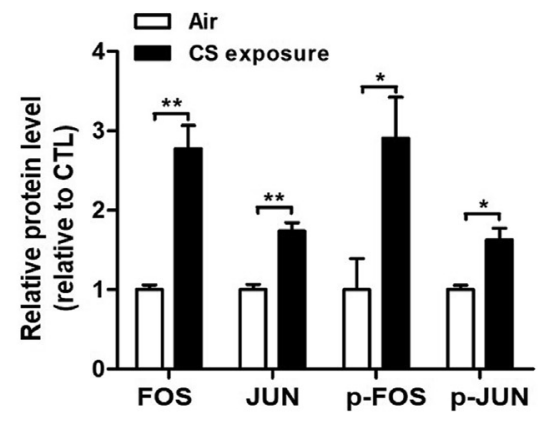

D

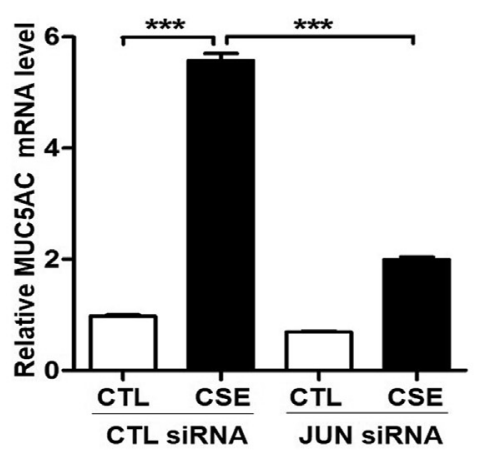

Figure 5 AP-1 mediates CS-induced MUC5AC hyperproduction. A: C57BL/6 mice were exposed to CS for 12 weeks. Lungs were isolated from individual mice 1 day after the last smoke challenge and were measured for the relative protein expression of AP-1. B: Quantification of the bands presented in A. C: Western blot analysis of the induction of AP-1 by CSE in human bronchial epithelial cells. Cells were treated with 3\% CSE for varying times from 0 to 9 hours before harvest. D and E: Human bronchial epithelial cells were pretreated with $5 \mathrm{nmol} / \mathrm{L}$ JUN siRNA or $10 \mu \mathrm{mol} / \mathrm{L}$ SP600125 (inhibitor of Jun amino-terminal kinase) and then were stimulated by 3\% CSE for 24 hours. MUC5AC mRNA level was assessed by qPCR. Data are expressed as means \pm SEM. $n=3$ independent experiments; $n=3$ CTL mice (A); $n=6$ mice for CS exposure (A). An unpaired two-tailed $t$-test was used in B. One-way analysis of variance followed by Newman-Keuls test was used in $\mathbf{D}$ and $\mathbf{E}$. ${ }^{*} P<0.05,{ }^{* *} P<0.01$, and ${ }^{* *} P<0.001$. AP-1, activator protein-1; CS, cigarette smoke; CSE, cigarette smoke extract; CTL, control; DMSO, dimethyl sulfoxide; $\mathrm{HBE}$, human bronchial epithelial; MUC5AC, Mucin 5AC; $\mathrm{p}-$, phosphorylated; qPCR, real-time quantitative PCR.
Thus, we hypothesized that ATF3-mediated CS-induced MUC5AC expression might be induced through AP-1. We established co-immunoprecipitation assays in CSEtreated HBE cells by using antibodies against FOS and p-FOS to determine whether ATF3 and AP-1 physically interact. The results showed that ATF3 and AP-1 coimmunoprecipitated with each other and that CSE could up-regulate ATF3 and AP-1, as well as the ATF3/AP-1 complex, in the nucleus (Figure 6, A and B). Moreover, we observed a large amount of ATF3 colocalized with AP1 in the nucleus of HBE cells after CSE exposure (Figure 6, C and D). To further elucidate the specific association of ATF3 with the MUC5AC promoter in airway epithelial cells, we used $\mathrm{HBE}$ cells to perform a $M U C 5 A C$ promoter-driven luciferase reporter assay. As shown in Figure $6 \mathrm{E}$, the up-regulation of $M U C 5 A C$ promoter activity by CSE was blocked by the ATF3 siRNA. Consistent with the mRNA expression data, the luciferase activity of MUC5AC was not affected by the overexpression of ATF3 (Figure $6 \mathrm{~F}$ ). To further verify that ATF3 was binding to the MUC5AC promoter via AP-1, a ChIP assay was performed with anti-ATF3 antibody. The data revealed that CS caused ATF3 to accumulate on the MUC5AC promoter (Figure $6 \mathrm{G}$ ). When the $A P-1$ gene was specifically knocked down by JUN siRNA, the interaction of ATF3 with the $M U C 5 A C$ promoter was reduced (Figure $6 \mathrm{H}$ ), suggesting that the binding of ATF3 to the MUC5AC promoter was mediated by AP-1.

\section{Discussion}

Various stimuli, such as chemicals, allergens, and bacteria, can increase mucus expression in airway epithelial cells. ${ }^{19}$ 
A

IP: FOS

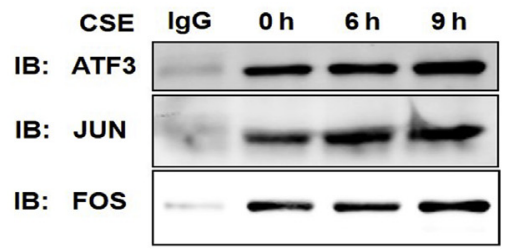

C

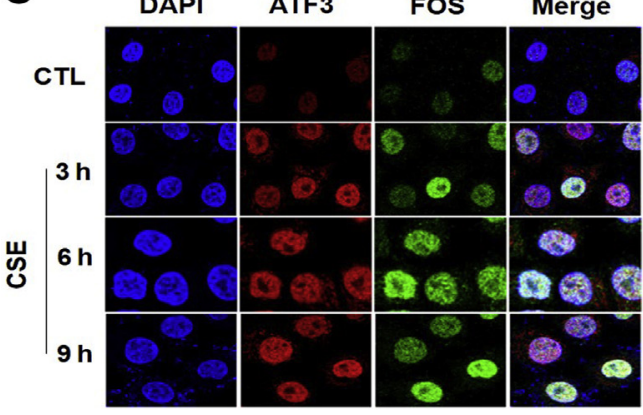

E

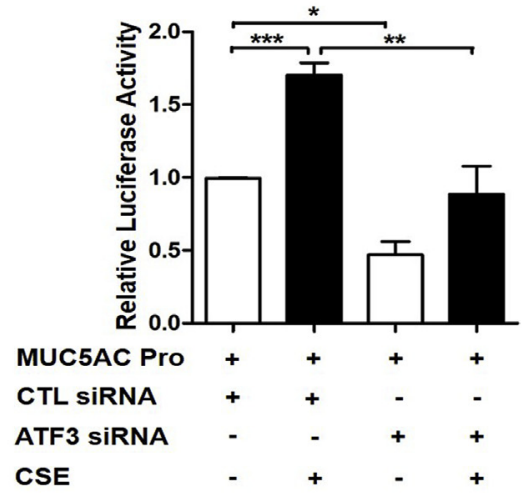

G

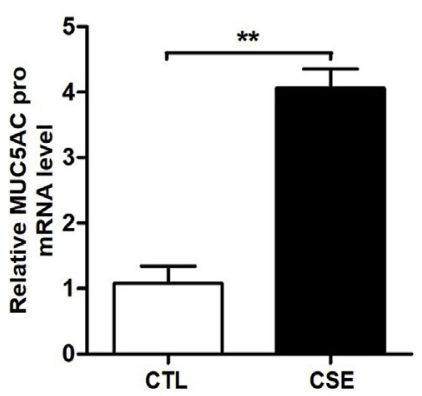

B

IP: p-FOS

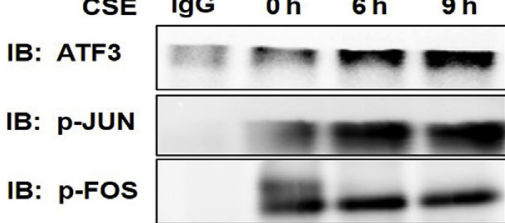

D

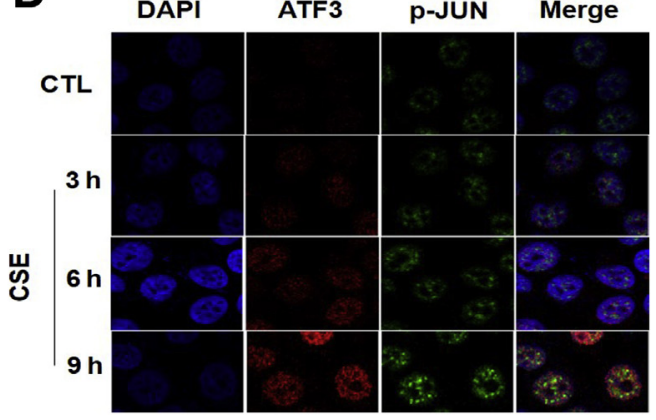

F

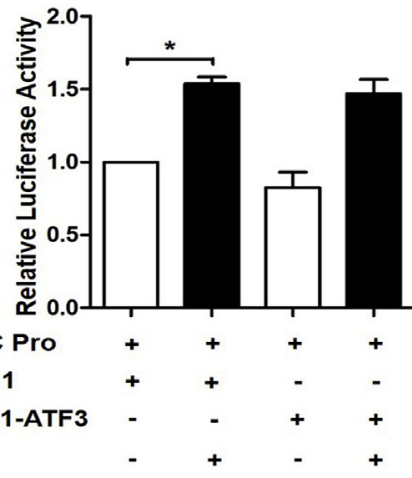

H

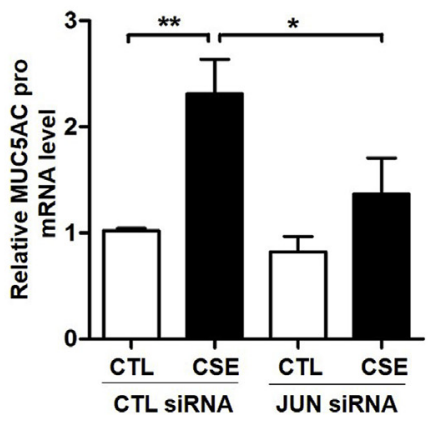

Figure 6 Regulation of CS-mediated MUC5AC production by ATF3 via AP-1-mediated signaling pathways. A and B: HBE cells were stimulated with 3\% CSE for varying times from 0 to 9 hours. Harvested nuclear protein was immunoprecipitated using control (IgG) or FOS or p-FOS antibodies and analyzed by Western blot analysis. C and D: Representative immunofluorescence images of ATF3 and AP-1 proteins colocalization after CSE exposure in HBE cells. DAPI counterstain was used to identify cell nucleus. E: HBE cells were pretreated with CTL or ATF3 siRNA and then transfected with the MUC5AC-reporter plasmid. Luciferase activity was then measured in CSE-treated and untreated cells. The transfections and luciferase assays were performed in triplicate. F: The control or ATF3 plasmid was cotransfected into HBE cells with the MUC5AC-reporter plasmid. Luciferase activity was then measured in CSE-treated and untreated cells. The transfections and luciferase assays were performed in triplicate. G: HBE cells were treated with 3\% CSE for 9 hours and were fixed with formaldehyde. Nuclear fractions were harvested and sonicated to generate nuclear lysate. Chromatin fragments were incubated with anti-ATF3 antibody. Eluted chromatin-ATF3 fragments underwent qPCR detection covering the MUC5AC promoter. H: After specifically knockdown of AP-1 protein, the binding ability of ATF3 to the MUC5AC promoter was weakened. Data are expressed as means \pm SEM. $n=3$ independent experiments. An unpaired two-tailed $t$-test was used in $\mathbf{G}$. One-way analysis of variance followed by Newman-Keuls test was used in $\mathbf{E}, \mathbf{F}$, and $\mathbf{H}$. ${ }^{*} P<0.05,{ }^{*} P<0.01$, and ${ }^{* * *} P<0.001$. AP-1, activator protein-1; ATF3, activating transcription factor 3; CS, cigarette smoke; CSE, cigarette smoke extract; CTL, control; HBE, human bronchial epithelial; IB, immunoblot; IP, immunoprecipitation; MUC5AC, Mucin 5AC; p-, phosphorylated; pro, promoter; qPCR, real-time quantitative PCR. 
Mucus overproduction causes restricted airflow, ultimately resulting in a loss of lung function. ${ }^{20}$ Considered to be an important characteristic of COPD, the suppression of mucus hypersecretion is a potential therapeutic target for chronic airway diseases. Therefore, this study focused primarily on CS-induced MUC5AC production. MUC5AC is a primary mucin gene that is expressed in the airway epithelium. As a result, we observed a significant increase of MUC5AC in human and murine airway epithelial cells after CSE treatment, which was similar to the results of a study performed by Schamberger et al. ${ }^{21}$ Moreover, in accordance with a previous work, ${ }^{22}$ we also observed mucus overproduction in the airway in a long-term CSexposed animal model.

As a member of the CREB/ATF family that participates in a wide variety of biological phenomena, ${ }^{23}$ ATF3 has been implicated in a wide range of cellular events, including apoptosis, and is associated with the transactivation of numerous genes. ${ }^{15}$ ATF3 can be induced rapidly by various stress signals, such as oxidative stress, elevated temperature, cytokines, hypoxia, DNA damage, and endoplasmic reticulum stress. ${ }^{14,24}$ In certain respiratory diseases, such as lung injury and allergic disease, ATF3 has been reported to play a protective role by inhibiting the production of various chemokines or cytokines and by preventing the recruitment of inflammatory cells. ${ }^{18,25,26}$ In allergic Atf $3^{-/-}$mice, more pronounced goblet cell hyperplasia and mucous hypersecretion were observed. ${ }^{18}$ However, little has been reported about the role of ATF3 in CS-related disease.

In this study, we first demonstrated that CS could up-regulate ATF3 transcript and protein levels in the pulmonary epithelium. We also observed an increased level of ATF3 in CS-exposed mice, suggesting that ATF3 might participate in CS-induced downstream signaling cascades. Interestingly, we observed that the knockdown of ATF3 significantly attenuated CS-induced MUC5AC expression, whereas the overexpression of ATF3 exerted no considerable effects. These data suggested that CSinduced endogenous ATF3 was sufficient to promote MUC5AC expression. However, it remains unclear why exogenous ATF3 failed to increase the basal levels of MUC5AC. One plausible explanation is that exogenous ATF3 might have lost its ability to bind to AP-1 and to promote MUC5AC expression.

Gilchrist et al ${ }^{18}$ identified ATF3 as a negative regulator of mucus hypersecretion in ovalbumin-treated mice, which is opposite to our finding in CS-exposed mice. These contradictory results related to mucus expression in asthma and COPD models may be due to the context-dependent activity of ATF3, and the difference might be ascribed to the different stress signals in these models, because the final readout or functional consequence will vary depending on the stress condition. ${ }^{12}$

Considering its emerging role in CS-induced MUC5AC expression, the potential mechanism of ATF3 action is an important issue to address. When present as a homodimer,
ATF3 usually acts as a negative regulator of gene transcription. However, when heterodimerized with other bZip proteins, such as members of the AP-1 family, CCAAT/ enhancer-binding proteins and growth arrest and DNA damage-inducible 153/CCAAT-enhancer-binding protein homologous protein 10, ATF3 can promote gene expression. ${ }^{24,27}$ The ATF3/JUN heterodimer has been demonstrated to play a role in cell cycle progression ${ }^{28}$ and heat shock protein 27 expression in neuronal cells. ${ }^{29}$ In our study, no direct ATF3 binding site was found in the -3724/3224 region of the MUC5AC promoter, but several AP-1 binding sites were identified. The transcription factor AP-1 could regulate the expression of genes related to the cellular stress response by associating with various proteins. ${ }^{30,31}$ Moreover, it has been reported that CS stimulation of AP-1 can activate the expression of the MUC5AC gene. ${ }^{16}$ Consistently, we observed increased production of AP-1 after CS exposure, whereas the targeted deletion of AP-1 conferred protection against CS-induced MUC5AC expression.

Because both ATF3 and AP-1 were involved in CSinduced MUC5AC production but only AP-1 had the ability to bind to the MUC5AC promoter, we proposed the hypothesis that after CSE stimulation the ATF3/AP-1 complex might exert a positive effect on MUC5AC transcription. Our co-immunoprecipitation analysis and

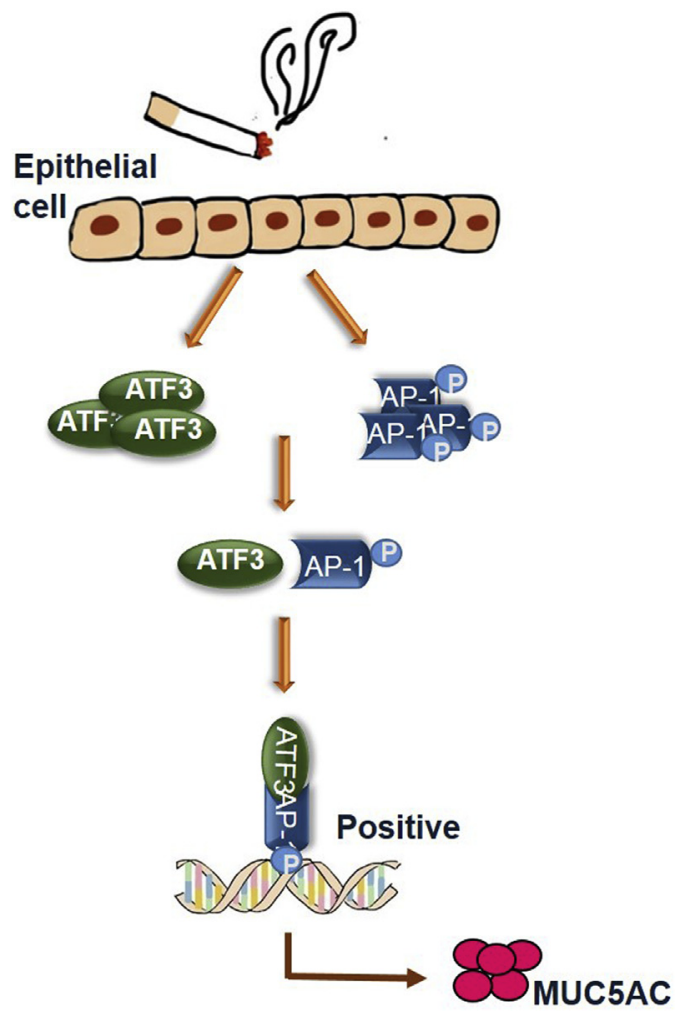

Figure 7 Schematic diagram representing regulation of CS-induced MUC5AC transcription via positive regulation by ATF3 dependent on AP-1 activation. AP-1, activator protein-1; ATF3, activating transcription factor 3; CS, cigarette smoke; MUC5AC, Mucin 5AC; P, phosphorylated. 
immunofluorescence colocalization analysis in HBE cells confirmed that AP-1 could form a heterodimer with ATF3 even under basal conditions. Phosphorylation of these AP-1-binding proteins can elevate their transactivation potential and thus promote AP-1-dependent gene transcription. ${ }^{16}$ Indeed, our luciferase assay and ChIP assay results revealed that CS-stimulated ATF3 expression could increase ATF3/AP-1 complex binding to the MUC5AC promoter and activate gene transcription. The deletion of the AP-1 protein weakened the ability of ATF3 to bind to the MUC5AC promoter, suggesting that this binding was mediated, at least in part, by AP-1. Thus, it was reasonable to speculate that CS induced MUC5AC expression in the airway epithelium by activating ATF3/AP-1 signaling pathway.

\section{Conclusion}

Our study demonstrates a potential role for ATF3 in the regulation of CS-stimulated MUC5AC expression and reveals the role of the ATF3/AP-1 complex in this process. Further investigations of ATF3-targeting therapies may help treat CS-induced mucus disorders (Figure 7).

\section{Acknowledgments}

We thank all of the study participants and their families.

Y.-p.W. and Y.-f.W. designed the experiments, performed the experiments, analyzed data, and wrote the manuscript; Z.-h.C., H.-h.S., and W.L. designed the experiments and co-wrote the manuscript; H.-b.Z., M.L., and C.Zha. provided materials for this study; C.Zhu and S.-m.Y. and C.C. checked the manuscript.

\section{References}

1. Pauwels RA, Buist AS, Calverley PM, Jenkins CR, Hurd SS; GOLD Scientific Committee: Global strategy for the diagnosis, management, and prevention of chronic obstructive pulmonary disease. NHLBI/ WHO Global Initiative for Chronic Obstructive Lung Disease (GOLD) Workshop summary. Am J Respir Crit Care Med 2001, 163: $1256-1276$

2. Lozano R, Naghavi M, Foreman K, Lim S, Shibuya K, Aboyans V, et al: Global and regional mortality from 235 causes of death for 20 age groups in 1990 and 2010: a systematic analysis for the Global Burden of Disease Study 2010. Lancet 2012, 380:2095-2128

3. Burgel PR, Martin C: Mucus hypersecretion in COPD: should we only rely on symptoms? Eur Respir Rev 2010, 19:94-96

4. Salvi SS, Barnes PJ: Chronic obstructive pulmonary disease in nonsmokers. Lancet 2009, 374:733-743

5. Shin IS, Shin NR, Park JW, Jeon CM, Hong JM, Kwon OK, Kim JS, Lee IC, Kim JC, Oh SR, Ahn KS: Melatonin attenuates neutrophil inflammation and mucus secretion in cigarette smoke-induced chronic obstructive pulmonary diseases via the suppression of Erk-Sp1 signaling. J Pineal Res 2015, 58:50-60

6. Rabe KF, Hurd S, Anzueto A, Barnes PJ, Buist SA, Calverley P, Fukuchi Y, Jenkins C, Rodriguez-Roisin R, van Weel C, Zielinski J; Global Initiative for Chronic Obstructive Lung Disease: Global strategy for the diagnosis, management, and prevention of chronic obstructive pulmonary disease: GOLD executive summary. Am J Respir Crit Care Med 2007, 176:532-555

7. Deshmukh HS, Shaver C, Case LM, Dietsch M, Wesselkamper SC, Hardie WD, Korfhagen TR, Corradi M, Nadel JA, Borchers MT, Leikauf GD: Acrolein-activated matrix metalloproteinase 9 contributes to persistent mucin production. Am J Respir Cell Mol Biol 2008, 38: 446-454

8. Vestbo J, Prescott E, Lange P: Association of chronic mucus hypersecretion with FEV1 decline and chronic obstructive pulmonary disease morbidity. Copenhagen City Heart Study Group. Am J Respir Crit Care Med 1996, 153:1530-1535

9. Vestbo J: Epidemiological studies in mucus hypersecretion. Novartis Found Symp 2002, 248:3-12; discussion 12-19, 277-282

10. Speizer FE, Fay ME, Dockery DW, Ferris BG Jr: Chronic obstructive pulmonary disease mortality in six U.S. cities. Am Rev Respir Dis 1989, 140:S49-S55

11. Lange P, Nyboe J, Appleyard M, Jensen G, Schnohr P: Relation of ventilatory impairment and of chronic mucus hypersecretion to mortality from obstructive lung disease and from all causes. Thorax 1990, 45:579-585

12. Hai T, Wolford CC, Chang YS: ATF3, a hub of the cellular adaptiveresponse network, in the pathogenesis of diseases: is modulation of inflammation a unifying component? Gene Expr 2010, 15: $1-11$

13. Hai T: The ATF transcription factors in cellular adaptive responses. Gene Expression and Regulation. Edited by Ma J. Beijing, China: Higher Education Press, 2006. pp. 329-340

14. Hai T, Wolfgang CD, Marsee DK, Allen AE, Sivaprasad U: ATF3 and stress responses. Gene Expr 1999, 7:321-335

15. Thompson MR, Xu D, Williams BR: ATF3 transcription factor and its emerging roles in immunity and cancer. J Mol Med (Berl) 2009, 87 : $1053-1060$

16. Gensch E, Gallup M, Sucher A, Li D, Gebremichael A, Lemjabbar H, Mengistab A, Dasari V, Hotchkiss J, Harkema J, Basbaum C: Tobacco smoke control of mucin production in lung cells requires oxygen radicals AP-1 and JNK. J Biol Chem 2004, 279:3908539093

17. Lam HC, Choi AM, Ryter SW: Isolation of mouse respiratory epithelial cells and exposure to experimental cigarette smoke at air liquid interface. J Vis Exp [Internet], 2011, 48:e2513. doi:10.3791/ 2513

18. Gilchrist M, Henderson WR Jr, Clark AE, Simmons RM, Ye X, Smith KD, Aderem A: Activating transcription factor 3 is a negative regulator of allergic pulmonary inflammation. J Exp Med 2008, 205: 2349-2357

19. Voynow JA, Rubin BK: Mucins, mucus, and sputum. Chest 2009, 135 $505-512$

20. Lai H, Rogers DF: New pharmacotherapy for airway mucus hypersecretion in asthma and COPD: targeting intracellular signaling pathways. J Aerosol Med Pulm Drug Deliv 2010, 23:219-231

21. Schamberger AC, Staab-Weijnitz CA, Mise-Racek N, Eickelberg O: Cigarette smoke alters primary human bronchial epithelial cell differentiation at the air-liquid interface. Sci Rep 2015, 5:8163

22. Bartalesi B, Cavarra E, Fineschi S, Lucattelli M, Lunghi B, Martorana PA, Lungarella G: Different lung responses to cigarette smoke in two strains of mice sensitive to oxidants. Eur Respir J 2005, $25: 15-22$

23. Mayr B, Montminy M: Transcriptional regulation by the phosphorylation-dependent factor CREB. Nat Rev Mol Cell Biol 2001, 2:599-609

24. Chen BP, Wolfgang CD, Hai T: Analysis of ATF3, a transcription factor induced by physiological stresses and modulated by gadd153/Chop10. Mol Cell Biol 1996, 16:1157-1168

25. Shan Y, Akram A, Amatullah H, Zhou DY, Gali PL, MaronGutierrez T, Gonzalez-Lopez A, Zhou L, Rocco PR, Hwang D, Albaiceta GM, Haitsma JJ, dos Santos CC: ATF3 protects pulmonary resident cells from acute and ventilator-induced lung injury 
by preventing Nrf2 degradation. Antioxid Redox Signal 2015, 22: $651-668$

26. Akram A, Han B, Masoom H, Peng C, Lam E, Litvack ML, Bai X, Shan Y, Hai T, Batt J, Slutsky AS, Zhang H, Kuebler WM, Haitsma JJ, Liu M, dos Santos CC: Activating transcription factor 3 confers protection against ventilator-induced lung injury. Am J Respir Crit Care Med 2010, 182:489-500

27. van Dam H, Castellazzi M: Distinct roles of Jun : Fos and Jun : ATF dimers in oncogenesis. Oncogene 2001, 20:2453-2464

28. Taub R: Liver regeneration 4: transcriptional control of liver regeneration. FASEB J 1996, 10:413-427
29. Nakagomi S, Suzuki Y, Namikawa K, Kiryu-Seo S, Kiyama H: Expression of the activating transcription factor 3 prevents c-Jun $\mathrm{N}$-terminal kinase-induced neuronal death by promoting heat shock protein 27 expression and Akt activation. J Neurosci 2003, 23: $5187-5196$

30. Lallemand D, Spyrou G, Yaniv M, Pfarr CM: Variations in Jun and Fos protein expression and AP-1 activity in cycling, resting and stimulated fibroblasts. Oncogene 1997, 14:819-830

31. Chinenov Y, Kerppola TK: Close encounters of many kinds: Fos-Jun interactions that mediate transcription regulatory specificity. Oncogene 2001, 20:2438-2452 